\section{Polypharmacy and clinical outcomes}

I read with interest the article by $\mathrm{Lu}$ and colleagues ${ }^{1}$ which shows that quality indicators of pharmacotherapy are associated with increased odds of admission to hospital, but reduced odds of death. However, some problems in the analysis may explain the results. Specifically, based on Tables 1 and 3, there are 15102 patients without use of drugs included in the analysis of the effects of potentially inappropriate medications (PIMs) and anticholinergic burden on the outcomes but not included in the analysis of polypharmacy. Because of the high mortality of these patients (91.6\%), they may not be representative of general older adults. If these patients are excluded, the mortality rates in patients without the use of PIMs or anticholinergics are much lower than those in the original reports $(24.6 \%$ v. $56.2 \%$ and $21.3 \%$ v. $41.9 \%$, respectively).

\section{Hsiu-Nien Shen MD}

Chi Mei Medical Center, Tainan City, Taiwan

\section{Reference}

1. Lu WH, Wen YW, Chen LK, et al. Effect of polypharmacy, potentially inappropriate medications and anticholinergic burden on clinical outcomes: a retrospective cohort study. CMAJ 2015;187:E130-7.

CMAJ 2015. DOI:10.1503/cmaj.1150048

\section{The authors respond}

We very much appreciate Shen's ${ }^{1}$ interest in our paper, ${ }^{2}$ however, Shen's comments are misleading.

First, Shen's recalculation of the number of patients who had a death event in Table 3, using data from Table 1, was incorrect. Our study is longitudinal, with up to 10 years of follow-up. Table 1 represents drug exposure at baseline and Table 3 represents exposure during follow-up. Therefore, direct calculation between these two tables is improper. Shen has recalculated the original data from Tables 1 and 3, and this approach results in a misinterpretation of our data.

Second, as our study is longitudinal with repeated measurements (up to
40 quarters), the event numbers of allcause and fracture-specific admissions could be larger than the patient numbers, meaning a patient could experience more than one all-cause or fracturespecific admission during the follow-up period. That is also the reason we adopt generalized estimating equation (GEE) models with an autoregressive correlation structure to fit our study design. The "no. of patients" in Table 3 is actually "no. of patients who experience a clinical event in an observational time unit" ( 3 mo in our study), under the framework of GEE models.

Wan-Hsuan Lu MS, Yu-Wen Wen PhD, Liang-Kung Chen MD PhD, Fei-Yuan Hsiao PhD

Graduate Institute of Clinical Pharmacy (Lu, Hsiao), College of Medicine, National Taiwan University, Taipei, Taiwan; Clinical Informatics and Medical Statistics Research Center (Wen), Chang Gung University, Taoyuan, Taiwan; Aging and Health Research Center (Chen), National Yang Ming University; Center for Geriatrics and Gerontology (Chen), Taipei Veterans General Hos ital; School of Pharmacy, College of Medicine (Hsiao); Department of Pharmacy (Hsiao), National Taiwan University Hospital, Taipei, Taiwan

\section{References}

1. Shen HN. Polypharmacy and clinical outcomes [letter]. CMAJ 2015;187:827.

2. Lu WH, Wen YW, Chen LK, et al. Effect of polypharmacy, potentially inappropriate medications and anticholinergic burden on clinical outcomes: a retrospective cohort study. CMAJ 2015; 187:E130-7.

CMAJ 2015. DOI:10.1503/cmaj.1150049

\section{Canadian Task Force obesity guidelines are unbalanced}

As an academic group of bariatric surgeons, we are disappointed that the Canadian Task Force guidelines ${ }^{1}$ did not balance the rather depressing evidence for the limited effectiveness of medical and lifestyle interventions in severe obesity, with the dramatic evidence in support of bariatric surgery. In appropriately selected patients, bariatric surgery is not only remarkably safe, but has the potential to achieve durable regression and remission of many obesity-related

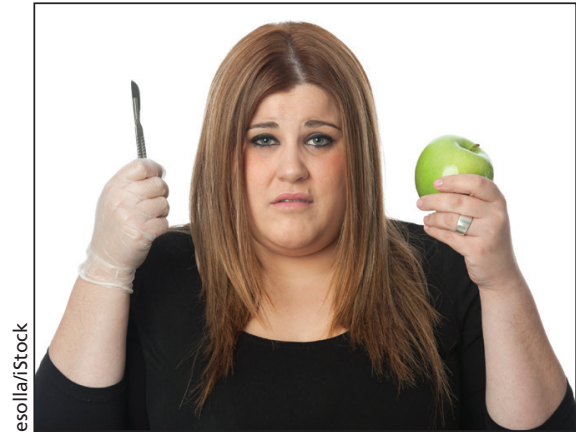

comorbidities such as type 2 diabetes, hypertension, sleep apnea, and even cancer. To not present a balanced picture of the care available to the obese patient is a disservice and to misrepresent the evidence for bariatric surgery in patients with severe obesity is unfortunate.

Chris de Gara MB MS, Aliyah Kanji

BSc MD, Shahzeer Karmali BSC

MPHMD, Dan Birch MSc MD

Department of Surgery (de Gara, Karmali), University of Alberta, Edmonton, Alta.; Division of General Surgery (Kanji), University of Alberta, Royal Alexandra Hospital, Edmonton, Alta.; Centre for the Advancement of Minimally Invasive Surgery (Birch), Alberta Health Services, Edmonton, Alta.

\section{Reference}

1. Brauer P, Connor Gorber S, Shaw E, et al. Recommendations for prevention of weight gain and use of behavioural and pharmacologic interventions to manage overweight and obesity in adults in primary care. CMAJ 2015; 187:184-95.

CMAJ 2015. DOI:10.1503/cmaj.1150050

\section{The authors respond}

We thank de Gara and colleagues' for their letter. ${ }^{1}$ Given the high prevalence of adult overweight and obesity in Canada, there was an urgent need to review evidence for primary care, the core focus of the Canadian Task Force on Preventive Health Care recommendations. ${ }^{2}$

We did not misrepresent the benefits of bariatric surgery: bariatric surgery is effective for the treatment of severe obesity and we did not state otherwise.

Rather, in the associated systematic treatment review in CMAJ Ppen $^{3}$ we specifically excluded bariatric surgery from consideration. 